\title{
Datos Estadísticos sobre la Preparación Sicoprofiláctica en el Equipo "Emesfao"*
}

Dres.: Carlos R. Silva Mojica, Miguel Fernández Bastidas, Hernando Amaya L., Belisario Calderón, Alberto Gómez T., Guillermo Lesmes, Guillermo López E. y Cecilia de Mojica

\section{CUADRO I}

ESTADISTICA GENERAL DEL EMESFAO

\begin{tabular}{|c|c|c|c|c|c|c|}
\hline Año & REUNION & No. Casos & Excelente & Bueno & Regular & M A L O \\
\hline 1957 & $\begin{array}{l}\text { III Congr. } \\
\text { Nal. Ob. Y } \\
\text { Gin. }\end{array}$ & 135 & $68.50 \%$ & $25-18 \%$ & $21-15 \%$ & $21-15 \%$ \\
\hline 1959 & $\begin{array}{l}\text { I Simposio } \\
\text { S. O. P. }\end{array}$ & $\begin{array}{l}311 \text { (1) } \\
\text { inc. ont. }\end{array}$ & $171-55 \%$ & $73 \cdot 12 \%$ & $34-11 \%$ & $69-22 \%$ \\
\hline 1960 & $\begin{array}{l}\text { I Simposio } \\
\text { s. P. O. }\end{array}$ & $\begin{array}{c}201 \text { (2) } \\
\text { no inc. ant. }\end{array}$ & $126-62 \%$ & $29 \cdot 14 \%$ & $21-100 / 0$ & $25-12 \%$ \\
\hline \multicolumn{2}{|c|}{ TOTALES } & 512 & $297-580 / 0$ & $66-13 \%$ & $55 \cdot 11 \%$ & $94-180 / 0$ \\
\hline
\end{tabular}

(1) Incluídos anteriormente.

(2) No incluídos anteriormente.

* Trabajo presentado por el "Equipo Médico de estudios sicofísicos de Analgesia Obstétrica de Bogotá" (EMESFAO) al II Simposio Colombiano de Sicoprofilaxis obstétrica. Mayo de 1960. Medellín. 


\section{CLASIFICACIONES}

Anterior

Actual

\section{EXCELENTE}

a) Paciente sin medicación sedante, analgésica o anestésica (salvo anestesia local para episiotomía).

b) Testimonio sobre ausencia de dolor o dolor tolerable.

c) Paciente consciente y lúcida en el nacimiento.
Parto a plena conciencia y sin dolor.

\section{BUENO}

a) Paciente que necesitó alguna analgesia.

b) Consciente en el nacimiento.
Dolor tolerable y lucidez perfecta durante todo el parto.

\section{REGULAR}
a) Dilatación indolora o con
Percepción de dolor controla- dolor tolerable.
ble; deseo de alguna analge-
b) Expulsión dolorosa. sia, quejas, alguna inquietud
c) Inconsciente en el naci- miento.

\section{MALO}
a) Trabajo doloroso (dil. y
Dolor intolerable. Inconscien- ex.)
b) Inconsciente en la dilata- te en el nacimiento.
ción y anestesiada en la ex- pulsión.

Todos los datos siguientes se refieren únicamente a los 201 casos presentados al II Simposio. 


\section{CUADRO II \\ EDAD DE LAS PACIENTES}

Oscila de 17 a 42 años: 1 caso de cada una de estas edades límites. Edad más frecuente: 23 años (22 casos)

\begin{tabular}{|c|c|c|c|c|c|c|c|c|c|}
\hline & & \multicolumn{2}{|c|}{ PARIDAD; } & & & & \\
No. pacientes & 85 & 50 & 29 & 18 & 8 & 4 & 2 & 1 & 4 \\
\hline Porcientaje & $\begin{array}{c}42.28 \\
\%\end{array}$ & $\begin{array}{c}24.87 \\
\%\end{array}$ & $\begin{array}{c}14.47 \\
\%\end{array}$ & $\begin{array}{c}8.95 \\
\%\end{array}$ & $\begin{array}{c}3.97 \\
\%\end{array}$ & $\begin{array}{c}2.01 \\
\%\end{array}$ & $\begin{array}{c}0.98 \\
\%\end{array}$ & $\begin{array}{c}0.46 \\
\%\end{array}$ & $\begin{array}{c}2.01 \\
\%\end{array}$ \\
\hline & & III & V & VI & VII & VIII & IX \\
\hline TOTAL Pacientes: 201 & 99.81 & $\%$ & & & \\
\hline
\end{tabular}

\section{EDAD DEL EMBARAZO}

9 meses: 187 casos $(93 \%)$

$\begin{array}{llll}81 / 2 & \prime & 10 & \text { casos } \\ 8 & , & 2 & (2 \text { malos }) \\ 71 / 2 & \prime & 2 & (2 \text { ex. })\end{array}$

CUADRO III

$N^{o}$ DE PARTOS CON P. S. P.

\begin{tabular}{|c|c|c|c|c|c|c|}
\hline No. de Partos & \multirow{2}{*}{ No. Pacientes } & $\%$ & \multicolumn{4}{|c|}{ R E S U L T A DO S } \\
\hline 10. & 154 & 77 & 92 & 22 & 16 & 24 \\
\hline 20. & 37 & 18 & 25 & 6 & 5 & 1 \\
\hline 3o. & 8 & 4 & 7 & 1 & & \\
\hline 40. & 1 & 0.5 & 1 & & & \\
\hline 5o. & 1 & 0.5 & 1 & & & \\
\hline
\end{tabular}


CUADRO IV

OCUPACION

Hogar : $185-92 \%$

Cabinera: 1 (Ex.)

Odontóloga: 1 (Ex.)

Profesora: 2 (Ex. y B.)

Oficinistas: 4 (Ex. todos)

Industrial: 1 (Ex.)

Sra. M. D.: 7 (5 Ex. 2 M.)

\section{EXAMEN SICOLOGICO}

Inestabilidad emocional. - Infantiles. - Temor al parto. Intento de suicidio. - Neurosis bélica. - Temor por complicaciones de partos anteriores. - Deseos de hacer sufrir al marido. Angustiadas. - Neuróticas. 34 pacientes: (17\%).

\section{RESULTADOS}

\begin{tabular}{|c|c|c|c|}
\hline $\mathrm{E}$ & $\mathrm{B}$ & $\mathrm{R}$ & $\mathrm{M}$ \\
\hline 12 & 7 & 5 & 10 \\
\cline { 1 - 3 } & & & \\
\hline
\end{tabular}

CUADRO V

\section{COMPLICACIONES DURANTE EL EMBARAZO}

Las historias registraron principalmente estas complicaciones: angustia; amenaza de aborto; parto prematuro; rechazo al embarazo; dermitis alérgica; hiperemesis; abandono del marido; anemia; rechazo al embarazo; muerte de un hijo; toxemia; $\mathrm{Rh}$ (-) ; grave mutilación a un hijo por un disparo; hidramnios; conflicto conyugal; grave enfermedad de la mamá; esterilidad de 5 años; afección renal; desprendimiento prematuro de la placenta a los 8 meses; hipertiroidismo. 
$\mathrm{N}^{\circ}$ de pacientes: $53(26.36 \%)$.

RESULTADOS

\begin{tabular}{|c|c|c|c|}
\hline $\mathrm{E}$ & $\mathrm{B}$ & $\mathrm{R}$ & $\mathrm{M}$ \\
\hline \multirow{2}{*}{29} & 8 & 5 & 11 \\
\hline
\end{tabular}

CUADRO VI

CALIFICACION DE LA PREPARACION

B: $173(86 \%)$.

$\mathrm{R}: 21$.

M: 7 (con un solo fracaso completo).

PREPARACION EXTEMPORANEA

\begin{tabular}{|l|c|c|c|c|}
\hline No. Casos & \multicolumn{4}{|c|}{ RESULTADOS } \\
\hline $111-5.470 \%$ & E & B & $R$ & $M$ \\
\hline & 5 & 2 & 1 & 3 \\
\hline
\end{tabular}

INDUCCION

\begin{tabular}{|l|c|c|c|c|}
\hline \multirow{2}{*}{ No. Casos } & \multicolumn{4}{|c|}{ RESULTADOS } \\
\hline \multirow{3}{*}{$33-160 / 0$} & E & B & R & M \\
\hline & 24 & 5 & 1 & 3 \\
\hline
\end{tabular}

CONDUCCION

$\mathrm{N}^{\circ}$ de casos: $50-25 \%$. 


\section{CUADRO VII}

\section{PRESENTACION}

OIA : 98 casos OIT: 15 casos OIP : 10 casos ODA : 21 casos ODT : 17 casos ODP : 19 casos. Pelvis : 7 casos (6 Ex. y 1 B.).

\section{ROTACION Y EXPULSION}

Rotación difícil en 28 casos $(14 \%)$ Variedades P. y T.

Expulsión difícil en 44 casos (22\%) Variedades P. y T. y Para I añosas.

\section{CUADRO VIII}

\section{DURACION DEL TRABAJO}

Duración del trabajo - Mayor frecuencia

\begin{tabular}{|c|c|c|c|}
\hline \multirow{2}{*}{ Primiparas } & Dilatación & Expulsión & Duración total \\
\hline & 7 horas & $25 \mathrm{~min}$. & $7 / / 2 \mathrm{~h}$. \\
\hline Multíparas & 5 & 15 & $51 / 2 \mathrm{~h}$. \\
\hline
\end{tabular}

Duración mínima: 1 hora -3 casos.

Duración máxima: 28 horas -1 caso.

\section{CUADRO IX}

\section{AMNIOTOMIA}

Fue practicada la amniotomía precoz en 107 casos. (53.45\%)

Episiotomías: $135-(67.16)\left\{\begin{array}{l}\text { Con anestesia: } 103 \\ \text { Sin anestesia: } 32\end{array}\right.$

Gesgarros perineales $\left\{\begin{array}{l}\text { Grado I: } 12 \\ \text { Grado II: } 2\end{array}\right.$ 
Suturas perineales: $149-4 \sin$ anestesia.

Aplicaciones de fórceps: $31(15 \%)\left\{\begin{array}{l}\text { Con anestesia } 22 \\ \text { Sin anestesia } 9\end{array}\right.$

Resultados: E. 13 - B. 7 - R. 5 - M. 6

Cesáreas: 7 casos.

Indicaciones : $\left\{\begin{array}{l}\text { Desproporción : } 4 \text { casos } \\ \text { Sufrimiento fetal: } 1 \text { caso } \\ \text { Primípara añosa: } 1 \text { caso } \\ \text { Para II con 1er. parto } 18 \text { años antes. } \\ \text { Pelvis 1 caso }\end{array}\right.$

Resultados E. 3 - B. 2 - R. 1 - M. 1.

\section{CUADRO X}

\section{OTRAS MANIOBRAS OBSTETRICAS}

Rotación difícil de la presentación: 5 casos.

Extracción podálica del $2^{\circ}$ gemelo: 1 caso (ex.)

Gran extracción podálica por procidencia del cordón: 1 caso (Ex.).

\section{DROGAS APLICADAS EN PERIODO DE BORRAMIENTO} (la mayoría en Para I)

En 30 pacientes $(14 \%)$ se aplicaron estas drogas:

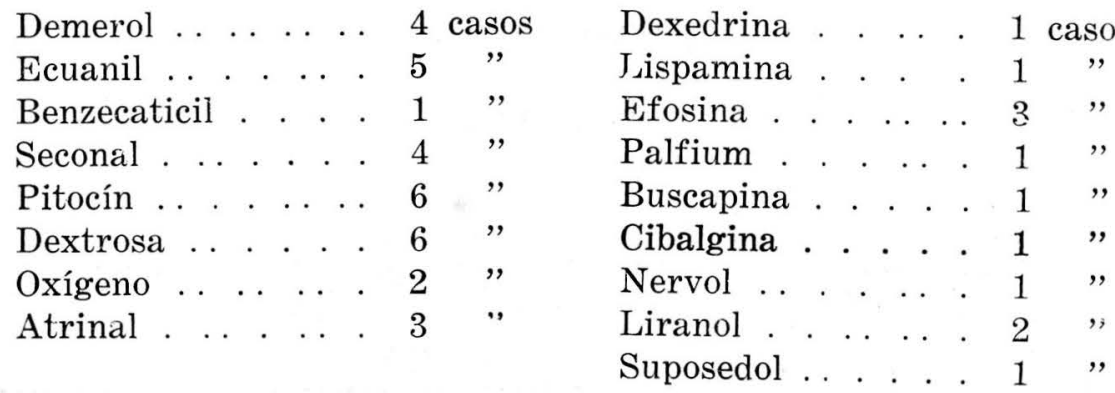




\section{CUADRO XI}

DROGAS APLICADAS EN PERIODO DE DILATACION: En 128 pacientes $(63 \%)$ :

\begin{tabular}{|c|c|c|c|c|c|c|c|c|c|}
\hline Dextrosa & $\therefore$ & 51 & casos & Buscapina & & & & & asos \\
\hline Pitocín & . & 63 & $"$ & Siquil & & & & & $"$ \\
\hline Oxígeno & . & 19 & , & Esparteína & & . & & & ", \\
\hline Demerol & 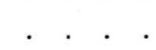 & 33 & $"$ & Ciclo .. & & & & & $"$ \\
\hline Seconal & & 2 & ", & Atrinal . & & & & & " \\
\hline Ecuanil & . & 13 & $"$ & Palfium . . & & . & & & $"$ \\
\hline Trilene .. & $\cdot$. & 2 & " & Esofina . & & . & & & ” \\
\hline Atarax & . & 3 & , & Atropina & & & & & " \\
\hline Conmel & & 1 & $"$ & Dexedrina & & & & 1 & $"$ \\
\hline
\end{tabular}

DROGAS APLICADAS EN PERIODO EXPULSIVO

En 124 pacientes $(63 \%)$

Oxígeno . . . . 84 casos

Dextrosa . . . . . 4 "

Pitocín . . . . 18 " Esparteína . . . 1 "

Demerol . . . . . 1 caso

(V. adelante tipo de anestesia).

\section{DROGAS APLICADAS EN EL POST PARTO}

Ergotrate a todas. Además en unos pocos casos (11): Pitocín (4), Dextrosa (7), Sangre (2), Veritol (2), Soludacortina $(1)$.

\section{CUADRO XII}

\section{ANESTESIA}

A. local . . $\ldots . \ldots . \quad \ldots 91$ casos

A. regional . . . 15 "

(B. silla y B. pudendo)

A. general $\ldots \ldots \quad \ldots \quad 50$

TOTAL .... 156 " 
TIEMPO DE ALUMBRAMIENTO

Tiempo mínimo

Tiempo máximo

Mayor frecuencia

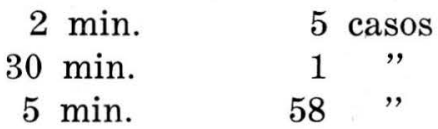

COMPLICACIONES ALUMBRAMIENTO

Extracción manual de la placenta 2 casos

Revisión uterina

7 (por hemorragia)

\section{CUADRO XIII}

DATOS DEL RECIEN NACIDO

(202 casos con un par de gemélos)

$\begin{array}{lllllllrr}\text { A término } \ldots & \ldots & \ldots & \ldots & \ldots & \ldots & 186 & 94 \% \\ \text { Prematuros } & \ldots & \ldots & \ldots & \ldots & \ldots & \ldots & 15 & 6 \%\end{array}$

Cuidados especiales del R. N.

(En 21 niños)

Oxígeno . . . . 20 casos Exsanguinotrans-

Incubadora . . . . 9 9

Analépticos . . . . 2 casos

APGAR (202 niños)

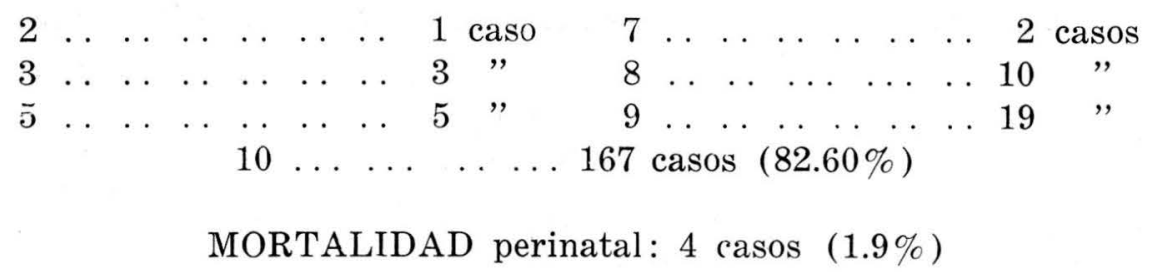

1 Muere a los 3 días por anomalía congénita intestinal.

2 Sufrimiento fetal. Asfixia M. M. (Apgar 3).

3 Desprendimiento de placenta (Apgar 2).

4 Brevedad absoluta del cordón. De término. Apgar 5. Murió al día siguiente. 


\section{CUADRO XIV}

\section{DESCONDICIONAMIENTO}

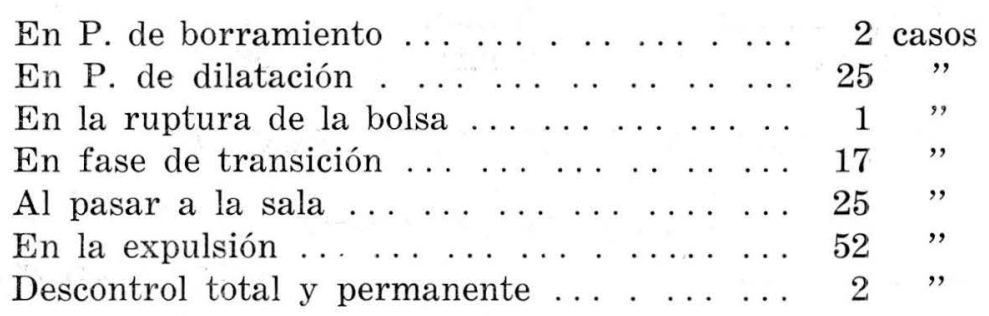

\section{CLASIFICACION DE RESULTADOS}

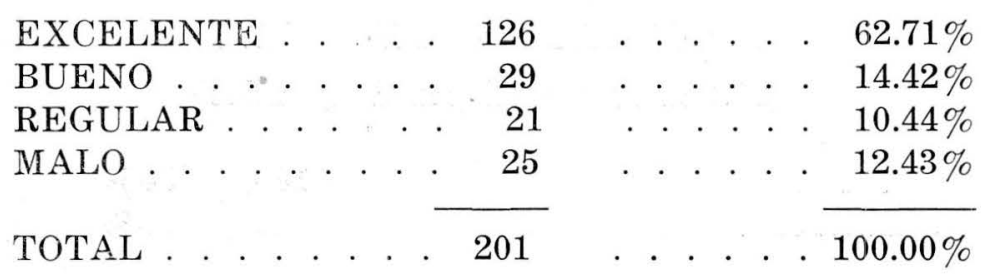

\section{RESUMEN Y CONCLUSIONES}

$1^{0}$ - El Equipo Médico de Estudios Sicofísicos de Analgesia Obstétrica (EMESFAO) presenta al II Simposio de Sicoprofilaxis obstétrica una estadística general sobre 512 casos, cifra muy significativa si se tiene en cuenta que se trata de pacientes particulares de los integrantes del Equipo.

2 ? - Presenta además una estadística detallada sobre 201 casos atendidos en la Clínica Palermo de Bogotá en el año de 1959 y principios del 60 .

$3^{\circ}$ - Las anotaciones han sido llenadas sobre el modelo de historia propuesta y adoptado por la Sociedad Colombiana de Obstetricia Sicosomática (SCOSS). Estas historias se encuentran en el archivo particular del Equipo.

$4^{\circ}$ - Del estudio comparativo de las distintas comunicaciones del Equipo se deduce que los resultados han ido mejorando año por año lo que indica más preparación en sus miembros, modificaciones favorables en el desarrollo y técnica de los cursos lo mismo que más confianza e información por parte del público respecto del método. 
$5^{0}$ - Se reconoce que el criterio de clasificación de resultados adoptado en el último período de estudio difiere del inicial, lo cual no basta para ensayar una comparación de estadísticas que de una idea aproximada de la realidad de los hechos.

$6^{\circ}$ - Se hace una lógica depuración de los casos excelentes pese al entusiasmo del obstetra tratante, para aplicar fielmente la clasificación de resultados aprobada en el primer Simposio, sin que esto mengue en lo mínimo los éxitos ni la bondad del sistema.

$7^{\circ}-$ El índice de fracasos del $12 \%$ tan solo, demuestra el interés del Equipo por perfeccionar la P. S. P. Se ha hecho un intento de estudiar las causas de fracaso, lo cual es motivo de una comunicación especial a este II Simposio.

$8^{\circ}$ - Ya es un lugar común anotar que la P. S. P. disminuye la duración del trabajo. En ello intervienen también otros factores: amniotomía precoz, inducción, conducción y posición de la paciente.

$9^{\circ}$ - Se hizo un amplio uso de sedantes y anestésicos mucho menor en todo caso que en pacientes no preparadas.

10 - Se anota esta vez un mayor uso de anestesia local y regional como de aplicaciones de fórceps sin anestesia y de episiotomías y episiorrafias sin la misma.

11 - Los resultados en el recién nacido son satisfactorios: Apgar 10 un $82 \%$ y mortalidad perinatal $1.9 \%$.

12 - El EMESFAO reconoce que ha dedicado poca atención a la preparación extemporánea de ahí el número reducido de casos presentados.

13 - Los integrantes del EMESFAO se muestran satisfechos de su labor, y como iniciadores del movimiento en el país, complacidos por la aceptacićn y contribuciones de que ha sido objeto hasta el presente por parte de muchos profesionales colombianos.

NOTA: En los casos clasificados como excelentes en este trabajo no se ha seguido un criterio estricto en cuanto a ausencia total de dolor ya que la mayoría de estas pacientes experimentaron un "dolor tolerable" y presentaron además un comportamiento organizado y ecuánime durante el parto. 